\title{
CLIPS based decision support system for water distribution networks
}

\author{
K. Sandeep ${ }^{1}$ and K. Rakesh ${ }^{2}$ \\ ${ }^{1}$ Research scholar, IIT Delhi, Delhi, India \\ ${ }^{2}$ Faculty, Department of Civil Engineering, Indian Institute of Technology, Delhi, India
}

Received: 7 February 2011 - Published in Drink. Water Eng. Sci. Discuss.: 7 March 2011

Revised: 8 September 2011 - Accepted: 23 September 2011 - Published: 27 October 2011

\begin{abstract}
The difficulty in knowledge representation of a water distribution network (WDN) problem has contributed to the limited use of artificial intelligence (AI) based expert systems (ES) in the management of these networks. This paper presents a design of a Decision Support System (DSS) that facilitates "on-demand" knowledge generation by utilizing results of simulation runs of a suitably calibrated and validated hydraulic model of an existing aged WDN corresponding to emergent or even hypothetical but likely scenarios. The DSS augments the capability of a conventional expert system by integrating together the hydraulic modelling features with heuristics based knowledge of experts under a common, rules based, expert shell named CLIPS (C Language Integrated Production System). In contrast to previous ES, the knowledge base of the DSS has been designed to be dynamic by superimposing CLIPS on Structured Query Language (SQL). The proposed ES has an inbuilt calibration module that enables calibration of an existing (aged) WDN for the unknown, and unobservable, Hazen-Williams C-values. In addition, the daily run and simulation modules of the proposed ES further enable the CLIPS inference engine to evaluate the network performance for any emergent or suggested test scenarios. An additional feature of the proposed design is that the DSS integrates computational platforms such as MATLAB, open source Geographical Information System (GIS), and a relational database management system (RDBMS) working under the umbrella of the Microsoft Visual Studio based common user interface. The paper also discusses implementation of the proposed framework on a case study and clearly demonstrates the utility of the application as an able aide for effective management of the study network.
\end{abstract}

\section{Introduction}

The day-to-day operation of a water distribution network is managed by skilled operators who use experience and other heuristics to adjust and control network elements such as pumps and valves in their desire to achieve satisfactory performance of the water supply system. However, following an unforeseen and an unusual emergency such as a burst water main, prompt and effective measures from the network manager is often taken for granted by the consumers. On their part, the network managers usually have to depend upon scant information that is reported by consumers impacted by these exigencies (Machell et al., 2010).

Correspondence to: $\mathrm{S}$. Kulshrestha (san1.djb@gmail.com)
Managers of water utilities design most of their interventions and other decisions based on intuition, rule of thumb heuristics and trial and error. These operative decisions are often vague and inconsistent as they lack objectivity and, as a result, these often result in inefficient network management. Further, on account of distribution complexities and excessive reliance on a heuristics driven management paradigm that is often unconvincing and seemingly feeble in substance without an accompanying validation, there is no guarantee that resources are utilized effectively (Raghvendran et al., 2007).

Difficulties in managing a typical water supply system arise on account of (i) absence of a well defined policy framework; (ii) difficulty in gathering information that is coherent and objective because, as is often the case, information is based on individual perception and experience; (iii) complexity of a typical water supply system on account of its extensive and spatially distributed character and accompanied by a variety of control mechanisms; and (iv) frequent 
changes in the network topology (Leon et al., 2000). Collectively, these foregoing issues present great difficulties in efforts to develop mathematical models of such inherently complex systems (Walski, 1993). Recent developments in AI technology make it possible to encode knowledge and reasoning in a structured computer program that enables it to mimic the human thought process and its application, albeit within a narrow but well defined domain specific framework, during a problem-solving process. Such a tool, generally referred to as either a knowledge-based system (KBS) or an Expert System (ES) (Chau, 2004), is designed to assist in solving problems that would otherwise require the skills and expertise of a knowledgeable "flesh and blood" professional by applying embedded heuristics and other pertinent rules of thumb.

The paper presents descriptive details of a DSS that has been developed to assist managers of WDN, especially where water supply is intermittent and demand driven and not pressure driven. Water distribution networks operate for a limited duration, and because the amount of water each user is able to collect depends on the available pressure at their connection and the duration of the service, the user demand is often not fully met. To improve the service standards, water utilities are struggling hard to develop aids in the form of computer based analysis and management software. Expert systems, as an artificial intelligence (AI) based approach, is one such approach that managers of water utilities are increasingly gravitating towards. The suggested tool is but a computer program that seeks to provide solutions to the same problems which were traditionally solved only by a human expert by archiving knowledge in the form of rules and other heuristics and then utilizing this pre-programmed set of strategies and the knowledge base in a manner that is akin to human reasoning. The AI field has always fascinated researchers who have applied it in many diverse fields (Cheng et al., 2002; Wang et al., 2004; Muttil and Chau, 2007). In the field of water management, some important applications of expert systems include EXPLORE (Leon et al., 2000), OASIS (Goforth and Floris, 1991), CRITQUING Expert System (Shepherd and Ortolano, 1996), IITWSEXP (Khosa et al., 1995), Expert System for treated water distribution (Bunn and Helms, 1999), Network Management System For Water Distribution System (Raghvendran et al., 2007), and Intelligent Control System For a Municipal Water Distribution Network (Chan et al., 1999). Most of these initiatives included components to transfer knowledge from the heuristic domain to the knowledge base of the expert system, while some of the other developments have applied fuzzy logic to process information and suggest "best practice" guidelines for the network manager.

Review of literature further reveals that presently available expert systems have an extremely limited scope and are confined to only those specific tasks that come as preprogrammed knowledge and are therefore unable to reason broadly across fields of peripheral expertise. These ES employ static knowledge domains without the ability to store newer facts and, therefore, are unable to learn and evolve. Moreover, the difficulty in knowledge representation of a WDN problem has contributed to the limited use of expert systems (ES) in the management of WDN. WDN are seldom new and are associated with large amount of data for its elements namely pipes, demand nodes, supply nodes and pump etc. As a result of the natural process of ageing, and with frequent interventions necessitated by our perceived need to meet various performance goals, water supply networks undergo physical changes that significantly impinge on their hydraulic responses. To analyze such a continuously changing system, it is necessary to store information related to WDN elements in a logical and systematic way, thereby preserving and retrieving the cross relations between them. The DSS, therefore, has been designed to have SQL as RDBMS.

\section{CLIPS expert shell and domain integration}

Knowledge base constitutes the core of any DSS and for the latter to be an effective surrogate for any expert, it is essential that the heuristic knowledge is available in the form of easily understandable rules. The public domain expert shell, CLIPS, provides an ideal platform wherein expert knowledge may be codified as rules related to operation and management of WDN. To supplement its rules management capability, CLIPS also works as an inference engine that enables it to perform the inference procedure whereby rules are interpreted to trigger various actions as is deemed appropriate. This mechanism employs the embedded pre-existing rules based knowledge as "facts" to drive the firing mechanism of the inference engine and thereby produce a recommended conclusion to a particular problem.

A feature of CLIPS is that this expert shell does not have its own dedicated database and, as a result, facts are volatile and are purged from its memory as soon its execution is terminated. To overcome this intrinsic limitation, an external RDMS must be added for a seamless integration with CLIPS. This then creates the desired two-way communication capability in the DSS whereby all facts can be transferred into the database during closing and, conversely, these facts can also be re-asserted back into the memory bank of CLIPS during program execution. In comparison to the previous efforts as documented in the literature, the structure and design of the proposed DSS is a significant improvement as it combines network modelling capabilities with ES. Hydraulic modelling requires complex and extensive calculations to be carried out and this capability has been achieved in the proposed DSS by integrating the MATLAB computation platform with the latter system. The present study attempts to develop a DSS that incorporates a dynamic knowledge acquisition system driven by simulated runs of a hydraulic model, suitably calibrated and validated for the given water utility. In addition to CLIPS as an AI tool and the 
MATLAB computational platforms, and in order to vest it with the capability to handle spatially distributed attributes of the study network, the proposed DSS uses open source GIS coupled together with a RDBMS. Together, these seemingly disjointed components work under the umbrella of a common user interface with cross platform integration being facilitated through a dynamic link library (DLL) in the Visual Studio environment.

\section{Problems of water distribution system management}

Municipal water distribution systems are: (i) spatially extensive; (ii) composed of multiple pipe loops to maintain satisfactory levels of redundancy; (iii) governed by non-linear hydraulic equations; (iv) designed complex hydraulic control devices such as valves and pumps; and (v) complicated by numerous layout, pipe sizing, and pumping alternatives. In addition to the palpable impairment in hydraulic performance that results as a network inevitably ages, it is natural that with a steady improvement in living standards accompanied by continuously increasing population, aspirations for better reliability in service and periodic inclusion of newer areas within the municipal limits especially in metropolitan cities together make these systems difficult to manage.

Often, these impacts result in performance levels that are well below expectations. Further, WDN are managed by experts, who, over the years of their association and responsibility, acquire an empirical knowledge of the system, but characteristically this knowledge remains largely confined to their respective personal domains. In the event of any new information and/or emergence of a new problem, these experts apply simple heuristics to design corrective measures and cognitively seek to predict network performance. Understandably, therefore, the assurance of a satisfactory response of the study network to suggested interventions is often based more on hope rather than on a validated belief.

With specific reference to issues of availability of domain knowledge, managers of water utilities face numerous difficulties on account of (i) expert scientific knowledge is not readily accessible; (ii) expert scientific knowledge is not available in a user friendly manner; and (iii) the tacit, undocumented expertise required for operation of a water supply system may be lost when experienced personnel leave service for whatever reason. These and similar concerns have seen resurgence in consumer demands that water engineers are suitably trained to apply current, technology based tools objectively and intelligently, and with the recent advances in AI technology, these personnel can be trained to fulfill this requirement. This indeed follows from the widely held view that there usually are very few specialists who have a thorough understanding of all the issues pertinent to network modelling for flows, pressures, and/or water quality. The proposed KBS has the potential to fill the gaps in knowledge that exist between researchers and practitioners (Chau, 2004).

Knowledge of basic network modelling concepts is indeed central to the decision-making process within water utilities not just as an aid to pipe network analysis but also for its efficient planning, design, and operation. This allows simulation of the design network and its examination under a variety of current and future operating conditions including possible design interventions. A number of off-the-shelf packages are available that allow tailor-made simulation models to be constructed for a water utility's specific requirements. Popular packages include EPANET (US Environmental Protection Agency), AQUIS (7T), Infoworks (Wallingford software), SynerGEE (Advantica), and WaterGEMS (Bentley).

These software packages enable development of mathematical models for a WDN that combine the physical configuration of these networks with physics-based equations that relate pressure and flow for each operational element (Machell et al., 2010). For the model to be acceptable as a reasonable representation of the actual system, the results from the model must bear close resemblance to the actual performance of the hydraulic system (ECAC, 1999). This, therefore, necessitates a prior model calibration.

\section{The DSS}

The aim of development of a DSS is to codify the heuristic expert knowledge in a form that is easily understood by the computer and also enables a novice to effectively manage a given WDN under unforeseen exigencies. Towards this end, possible real world scenarios corresponding to various design interventions are generated and corresponding results are incorporated in the knowledge base in order to enhance the scope and reach of the DSS. The development process involves (i) understanding the physical component elements of a typical WDN and their functionality, (ii) study of already existing DSS, (iii) understanding common terminology used by operators and experts, (iv) knowledge of current strategies for management of a water utility.

Since the DSS is to be applied on a WDN, it is imperative to have a modal case study of a WDN. Anytown Network (Walski et al., 1988; Kapelan, 2002), as shown in Fig. 1, is taken as the model case study. The network consists of 35 pipes assembled in a fashion that they form 19 loops. As the present study deals only with steady state analysis, the tanks at nodes 501 and 502 in the original network have been changed to reservoirs.

The proposed DSS is developed as a personal computer (PC) based application in the Visual Studio.Net environment. Visual Studio.NET is a complete set of development tools for building ASP Web applications - XML Web services, desktop applications, and mobile applications, etc., and all use the same integrated development environment (IDE), which allows them to share tools and facilitates in the creation of 


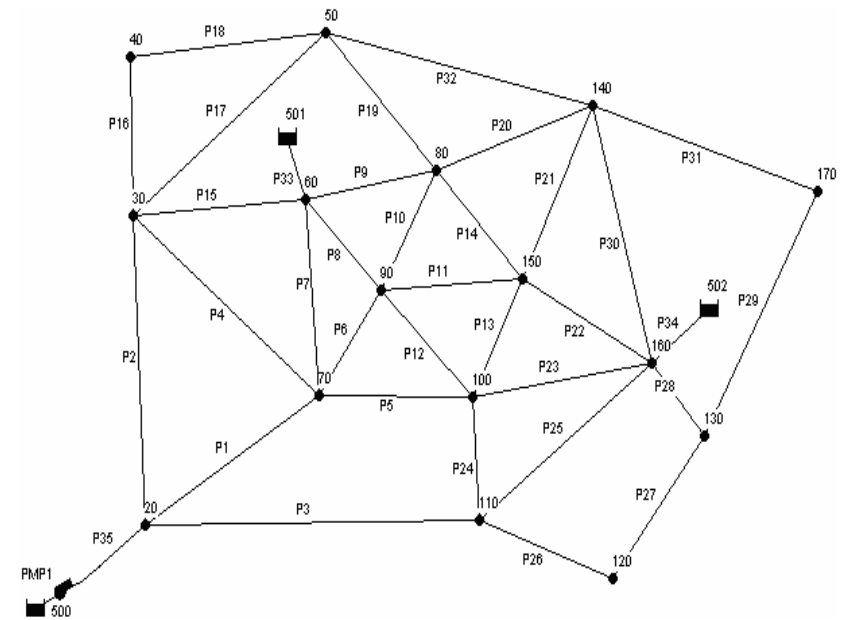

Figure 1. The anytown network.

mixed-language solutions. This framework provides a clear, object-oriented, extensible set of classes that enables one to develop rich Windows applications.

A DSS framework for a system as spatially extensive as a WDN has to reflect the underlying complexity of the system for realism and yet be simple to use. Accordingly, the complex functionality of these and similar systems is best disaggregated into a limited number of manageable facets in the form of interconnected modules and linked together in a structured manner to yield the overall DSS. Accordingly, within this modular framework, the proposed DSS has been designed with object modules for Network Calibration, Network Simulation, Daily Run Operation, and Network Information. The complex task of computations, data handling, visualization, and decision/inference of each module is handled through MATLAB, SQL server, MapWinGIS, and CLIPS. Figure 2 shows that platforms such as SQL, MATLAB, CLIPS, and MapWinGIS enable exchange/transfer of data between different modules of the DSS under the overall umbrella of Visual Studio.Net platform. In addition to the modules, there are additional supporting tools that have been integrated within the expert DSS. These supporting tools are listed in the following text along with a brief accompanying description.

\subsection{Relational database management system}

A typical WDN may be characterized in terms of a diverse set of attributes and, importantly, these attributes are complimentary in nature with no attribute capable of acting as a surrogate for one or more of the other identified attributes. While some attributes are static in nature (for example link diameters and elevations of the identified critical elements of the distribution system), many of the other attributes are dynamic in nature and may assume different values through time. The skill with which these data are stored, retrieved,

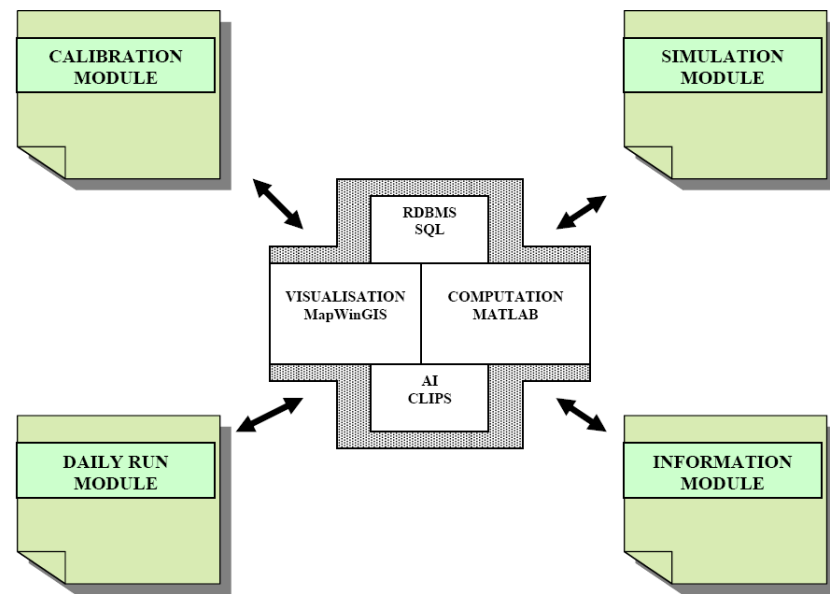

Figure 2. Basic framework of Decision Support System.

and/or used largely determines the utility and acceptability of any proposed DSS.

Typically, data pertaining to the components of WDN must be collected from different sources and need to be processed and stored within a well-structured framework in order to ensure availability to modellers, planners, and decision makers on demand and, at the same time, amenable to easy discrimination by the processing system. Relational databases allow such interrelationships to be preserved within the overall database management framework.

In the present study, a relational database management system (RDBMS) for WDN has been developed using Structured Query Language (SQL). All the necessary tables have been created in SQL and these are linked to each other through a primary key.

\subsection{CLIPS knowledge base and inference engine}

CLIPS (acronym for C Language Integrated Production System) is a computer language designed and developed by the US Space Agency, NASA, specifically for writing expert system (ES) applications. CLIPS is a forward-chaining rule-based language that resembles OPS5 and ART, other widely known rule-based development environments. Figure 3 shows the basic components of CLIPS and the basic functionality of each of these components is given as below:

1. User Interface: the mechanism by which the user and the ES communicate.

2. Fact-list: a global memory for data inserted as pipefacts, node-facts, valve-fact, etc. into the memory of CLIPS.

3. Knowledge-base: contains all the rules used by the ES. 


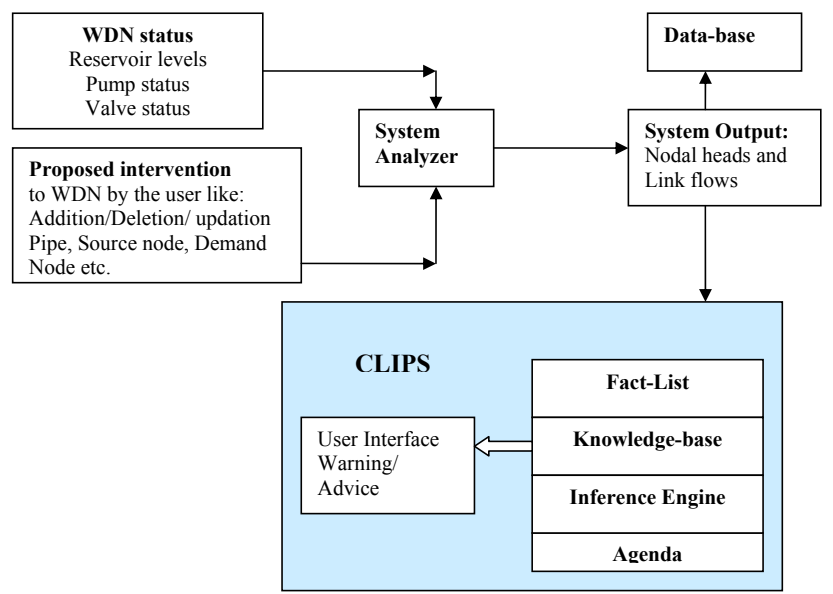

Figure 3. Components of CLIPS and its implementation in ES.

4. Inference engine: makes inferences by deciding which rules are satisfied by facts, prioritizes the satisfied rules, and executes the rule with the highest priority.

5. Agenda: a prioritized list created by the inference engine of instances of rules whose patterns are satisfied by facts in the fact-list.

CLIPS is a fact driven environment where rules are fired depending upon the pattern matching. CLIPS does not have its own database and facts stay in the memory of the CLIPS during its active state only. As soon as the CLIPS environment is closed, all facts are removed from its memory. Therefore, a mechanism was required through which facts are inserted into the memory from the database and viceversa. This was achieved through creation of classes and sub-classes in Visual Studio environment. CLIPS has been integrated with Visual Studio through DLL. CLIPS is fact driven language that works on pattern matching mechanism of the facts. It is therefore necessary to insert facts based on a certain template only. In CLIPS, the "deftemplate" construct is used to create a template which can then be used to access fields by name. Main class of all facts is "Fact". To facilitate transfer of data from CLIPS to Visual Studio and vice-versa in particular template, only two child classes have been created for each of the objects as pipe, source node, demand node, etc. Figure 4 shows two child classes for pipe object of WDN. First child class ("DRAFPipe") captures the value of each of the slots ("pipe_id", "snode", etc.), as shown in Fig. 4, values from database or user through user interface. Second child class ("DRPipe") saves the captured values in the format defined by template.

The chaining mechanism of CLIPS is programmed to fire rules if the inserted fact matches any of the already existing facts. Further, for CLIPS to become operational as an ES shell, the following two kinds of integration are required. The interface required for such an integration to be possible

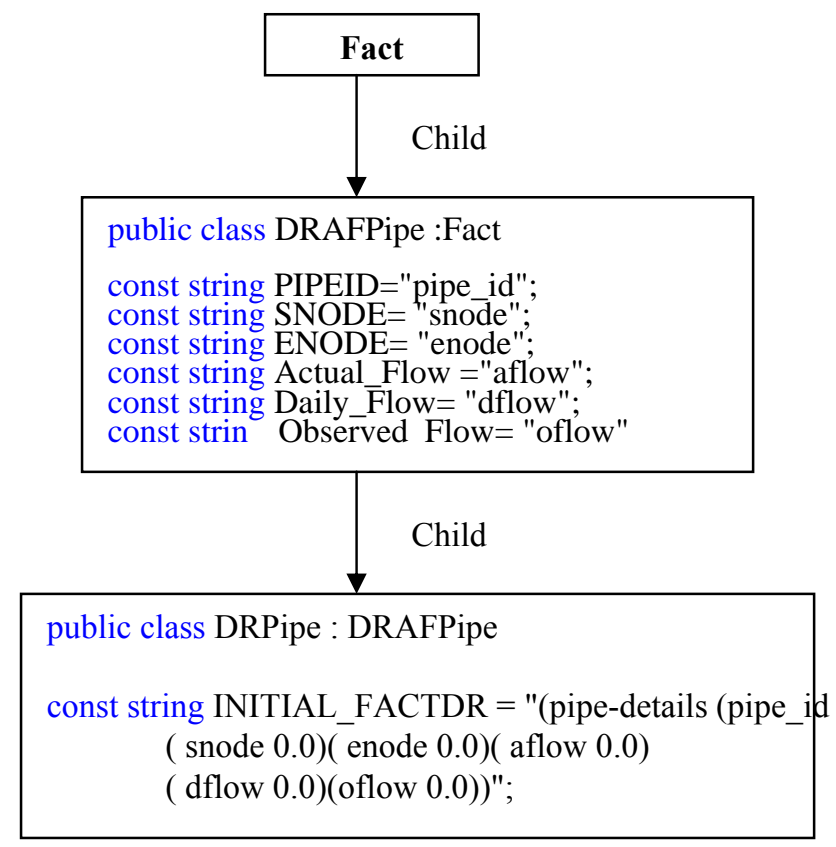

Figure 4. Class and child Class for fact of Pipe object.

enable (i) CLIPS to be embedded in other software platforms, and (ii) calling external functions from CLIPS. In the present study, integration of CLIPS within the Visual Studio.Net platform has been accomplished through ClipsNet.dll.

\subsection{MATLAB platform}

CLIPS and SQL server are not vested with the capability to perform complex mathematical computations. Therefore, in order to empower the DSS with computational capabilities, the MATLAB computing engine (acronym for MATrix LABoratory) has been integrated with the DSS in this study. The MATLAB engine incorporates the LAPACK and BLAS libraries, which provide access to state-of-the-art in software standards for matrix computation. The proposed DSS uses standalone COM components created using the MATLAB compiler. Each of the COM components is used in the Visual Studio.Net platform as a class that can perform mathematical operations on data imported from SQL and restore derived results back to the ".Net" environment.

\subsection{Hydraulic network solver}

The possibility of linking public domain network solver, EPANET (Rossman, 1993), through DLL was examined and it was felt that an inbuilt solver would be economical in terms of computer time. Therefore, based on the Todini and Pilati (1988) improved gradient method, a hydraulic network solver was developed in MATLAB and incorporated in Visual Studio.Net through a COM component. 


\subsection{Open source GIS platform}

MapWinGIS is an open source GIS and an application programming interface (API) distributed under the Mozilla Public License (MPL) that was built upon the Microsoft.Net Framework 2.0. The MapWinGIS components and end user application support manipulation, analysis, and viewing of geo-spatial data in many standard GIS data formats. Hence, MapwinGIS is a mapping tool, a GIS modelling system, and a GIS API in a re-distributable open source.

Further, and importantly, the creation, visualization, and manipulation of the shape files of various constituent elements of the study network have been achieved through integration of "ax-Map object" of MapWinGIS with Visual studio.Net.

\section{DSS modules}

The DSS being developed has modules on calibration, simulation, planning, and daily run.

\subsection{Network calibration module}

Various automatic network calibration algorithms have been proposed in the literature and include: (i) methods based on analytical equations (Walski, 1983); (ii) simulation models (Boulos and Ormsbee, 1991; Gofman and Rodeh, 1982; Ormsbee and Wood, 1986; Rahal et al., 1980); and (iii) optimization methods (Coulbeck and Orr, 1984; Ormsbee, 1989). Techniques based on analytical equations require significant simplification of the network through skeletonizations. As a result, such techniques may only get the user close to the correct results. Conversely, both simulation and optimization approaches take advantage of using a complete model. In the recent past, the focus of researchers has shifted from traditional analytical techniques to heuristic techniques such as Genetic Algorithm (GA), Simulated Annealing (SA), Shuffled Frog-Leaping Algorithm (SFLA), and Ant Colony Optimization (ACO). Performance of ACOA, as a class of optimization techniques, has been quite encouraging and this technique has often been seen to outperform other heuristicsbased approaches when applied to some benchmark problems (Maier et al., 2003; Zecchin et al., 2005).

Considering that the calibration process involves adjustment of some key system parameters like nodal demands and/or pipe-roughness values, prior knowledge of their rational values can act as a heuristic in stochastic search procedures such as GA and ACOA. In this study an automated calibration algorithm was developed for WDN and has been incorporated in the proposed DSS. The calibration algorithm has been designed using ACO approach and searches for optimal hydraulic performance by adjusting internal pipe roughness as a calibration parameter. As an aid, the algorithm has been designed to use age of the network pipes as prior information (PI) for guiding the search (Kulshrestha et al., 2011).

Accordingly, and in line with the foregoing discussion, a Calibration Module has been developed as an intrinsic component of the DSS. The calibration process has been developed based on ACO algorithm and systematically adjusts the Hazen Williams C-values in order to tune the hydraulic performance of the model, as expressed in terms of the derived distribution of nodal heads and link flows, with the network observations on corresponding state variables. The use of prior information (PI) on age and diameter of the various network components has been studied extensively in the study and is shown to aid the calibration process significantly. A detailed discussion on the development of the ACO-based calibration algorithm along with its evaluation and detailed sensitivity analysis is described separately in (Kulshrestha et al., 2011). The paper also presents a comparison of its performance with that of a MATLAB-based implementation of GA-based algorithm as well as the industry standard DARWIN CALIBRATOR of Benteley Systems, USA.

The significance of the role of some of the key parameters of the designed calibration algorithm in the calibration process has been studied extensively and their respective significance established on the basis of a detailed parametric study as reported in (Kulshrestha et al., 2011). The calibration algorithm parameters that have been the subject matter of scrutiny in the latter study include (along with their respective derived best values): (i) decision control parameters ( $\alpha=1.0$ and $\beta=0.3$ ); (ii) initial pheromone value, $\tau_{0}=200$; (iii) pheromone persistence factor, $\rho=0.95$; (iv) pheromone Reward factor, $R=200$; (v) number of ants, $m=10$; and (vi) maximum number of iterations, $n=200$.

Basic working of the calibration module is shown in Fig. 5. Observations on the state variables of link flows $(Q)$ and nodal heads $(H)$ at different loading conditions are taken as input parameters. The study network is calibrated using ACOA with one set of observed boundary conditions and then validated with another, but independent, set of boundary conditions. The calibrated network is saved in the RDBMS for scenario generation in response to various unforeseen, but hypothetical, design interventions that may be necessitated in the foreseeable future.

\subsection{Simulation module}

A typical network simulation model is comprised of static asset information such as pipe lengths, diameters, connectivity, and network topography. In addition to the static information, the model is also armed with information about dynamic attributes that are expected to change with time. These include distribution of water demand across the network and, importantly, direct or indirect measures of growth in pipe roughness with age, amongst others. Most contemporary modelling packages utilize models that include every pipe down to the level of, but not including, customer 


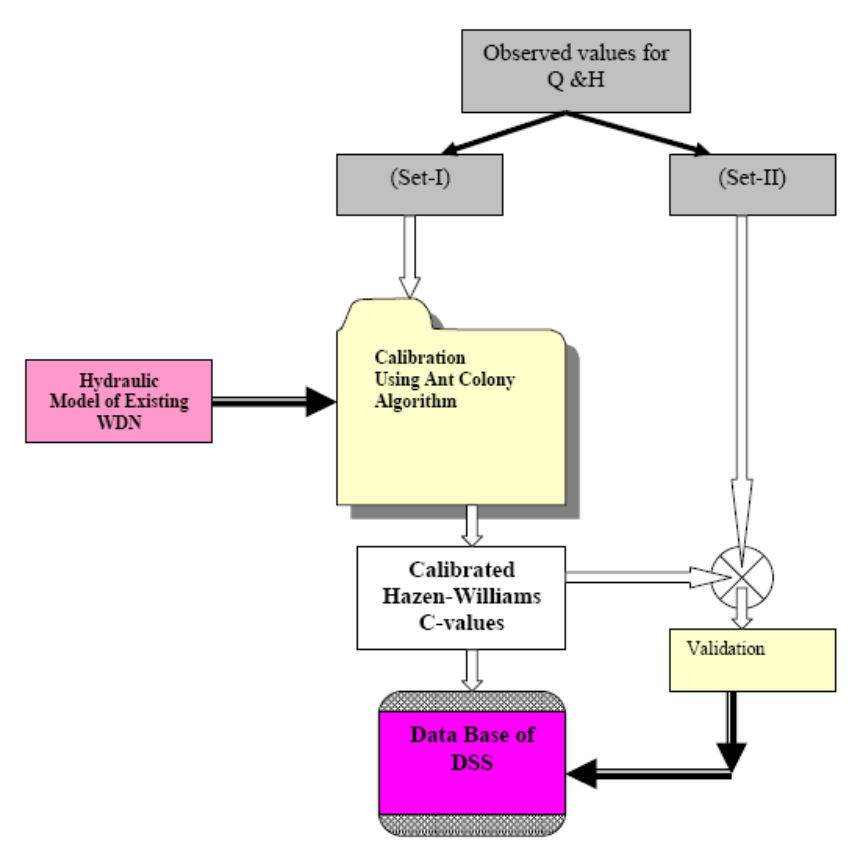

Figure 5. Schematic of calibration module.

service pipes. Standard daily time-varying demand relationships are estimated for different customer types, and these are summed and allocated at pipe junctions (nodes) according to the distribution of customers. Simulation models are normally used to simulate flows and pressures over a fixed $24 \mathrm{~h}$ period (Machell et al., 2010) and are usually vested with the capability to generate a great number of reports. The variety of data generated sometimes makes analysis and decision making tedious.

The simulation module of the proposed DSS is designed to enable simulation studies for planning enhancements/upgrades for the WDN as well as solving network problems. To meet service standards for a growing and increasingly fastidious population, it becomes imperative to add newer areas under the coverage of the distribution network or, in some situations, either add a new parallel link or even replace an existing smaller diameter pipe with a larger diameter pipe. A simulation study on WDN can examine the impact of any proposed or even a hypothetical intervention and, upon recall from the memory bank of the DSS, can guide the network managers whenever similar interventions are deemed to be desirable in future.

Figure 6 shows the schematic of the simulation module. It merits mention that a suggested change in the network configuration constitutes a valid scenario and may already exist in the knowledge base after having been generated following a prior query. In such a case, the user has the option to either evaluate the scenario details or, in the event the information is not already present in the knowledge base in the absence of any past interest in such a scenario, the network solver is triggered for flows and nodal heads. The values of pipe flows

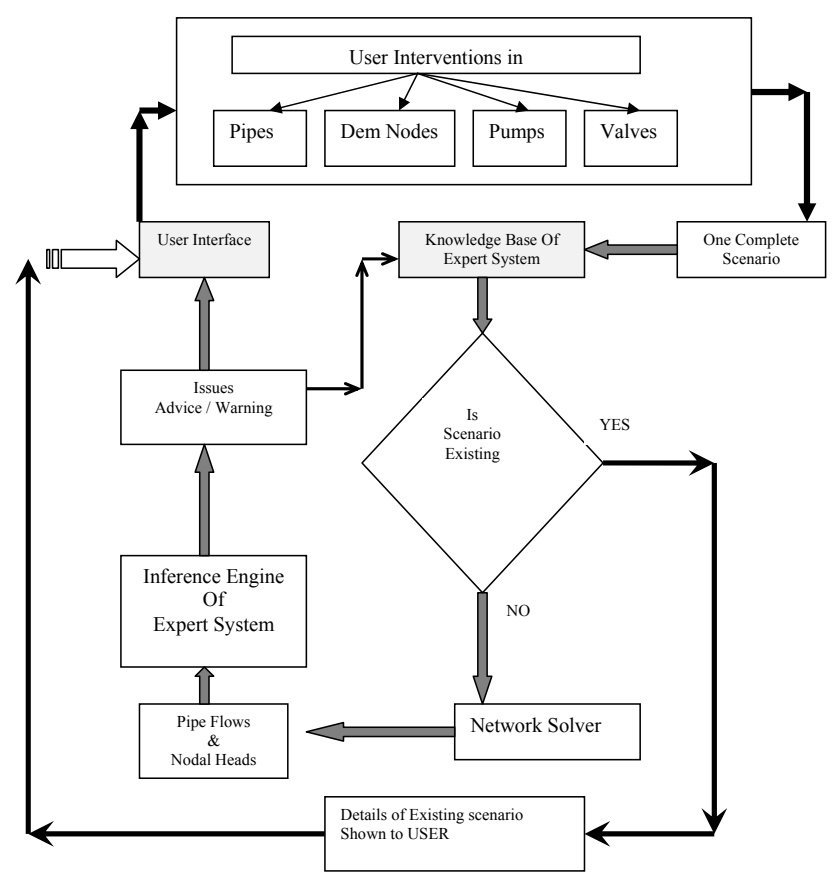

Figure 6. Schematic of simulation module of the DSS.

and nodal heads are asserted as facts in inference engine of CLIPS, which fires certain rules to issue Advice/Warning to the user. The Advice/Warning along with its corresponding scenario is added to the Knowledge Base of the DSS as an upgrade for future reference, thus rendering the DSS as dynamic and amenable to upgradation/augmentation. In order to facilitate delivery of existing (previous memory) and current (user interventions) information, two templates, namely "memory" and "user", are created in CLIPS.

\subsection{Planning module}

Planners carefully research all aspects of a water distribution system and try to determine which major capital improvement projects are necessary to ensure the quality of service for the future. This process, called master planning (also referred to as capital improvement planning or comprehensive planning), may be used to project system growth and water usage for the next 5, 10, or 20 years. System growth may occur because of population growth, annexation, acquisition, or wholesale agreements between water supply utilities (Walski et al., 2001).

The ageing of pipes, and a consequent increase in internal pipe roughness, is always accompanied by loss of conveyance. Such a scenario is indeed realistic and confronts all water supply networks. In water utility practices, HazenWilliams C-factor is the most commonly used parameter to represent internal roughness of water mains and by extension their carrying capacity and internal roughness of water mains (Walski et al., 1988), and the present study has also adopted this approach. The pipe roughness grows roughly linearly 
with time and the rate of roughness growth, a, is strongly influenced by the $\mathrm{pH}$ of water (For CI pipes).blackboxbf sentence break created here The corresponding model for growth of roughness may be written as (Colebrook and White, 1937):

$e=e_{o}+a t$

In Eq. (1), $e$ is the absolute roughness height, $(L) ; e_{0}$ is the initial roughness height, $(L) ; a$ is the growth rate in roughness height, $(L / T)$; and $t$ is the time variable, $(T)$. Using Colebrook and White (1937) and Swamee and Jain (1976) approximations, Hazen-Williams $C_{\mathrm{HW}}$ may respectively be written as (Walski et al., 1988).

$C_{\mathrm{HW}}=18.0-37.2 \log (X)$

$C_{\mathrm{HW}}=33.3|\log (0.27 X)|^{1.08}$

where $X=\left(e_{0}+a t\right) / D$ and $D$ is the diameter of pipe. The relationship between the annual roughness growth rate $(a)$ and the Langelier saturation index (LI), with the latter controlled by factors such as the $\mathrm{pH}$ of water, its alkalinity, and calcium content, can be represented as Eq. (4) (Lamont, 1981):

$a=10^{-(4.08+0.38 L I)}$ for LI $<0$

Given the age of the pipe and its diameter of old pipe, it is possible to obtain an estimate of the Hazen-Williams Cfactor. Based upon the above theoretical account, as the system undergoes the ageing process, the following things are expected to happen for any WDN:

1. With age of the WDN, the Hazen-Williams C-value will reduce.

2. The consequent head loss in the WDN will increase.

3. The total energy requirement in the WDN for the same input parameters will as well increase.

Therefore, from the management perspective, it is important to be forewarned on these changes and, at the same time, know in advance the manner in which its consequences are likely to manifest. As an example, the proposed planning module estimates Hazen-Williams C-values expected over various time horizons of 5, 10, 15, and 20 years and, for each such horizon, additionally computes (i) total energy requirement, (ii) total head loss across the network, (iii) head loss in each individual pipe, and (iv) temporal variation of HazenWilliams C-values.

The module further has the capability to present a graphical depiction of the above information besides populating the databases of the proposed expert DSS. The schematic of planning module is shown in Fig. 7.

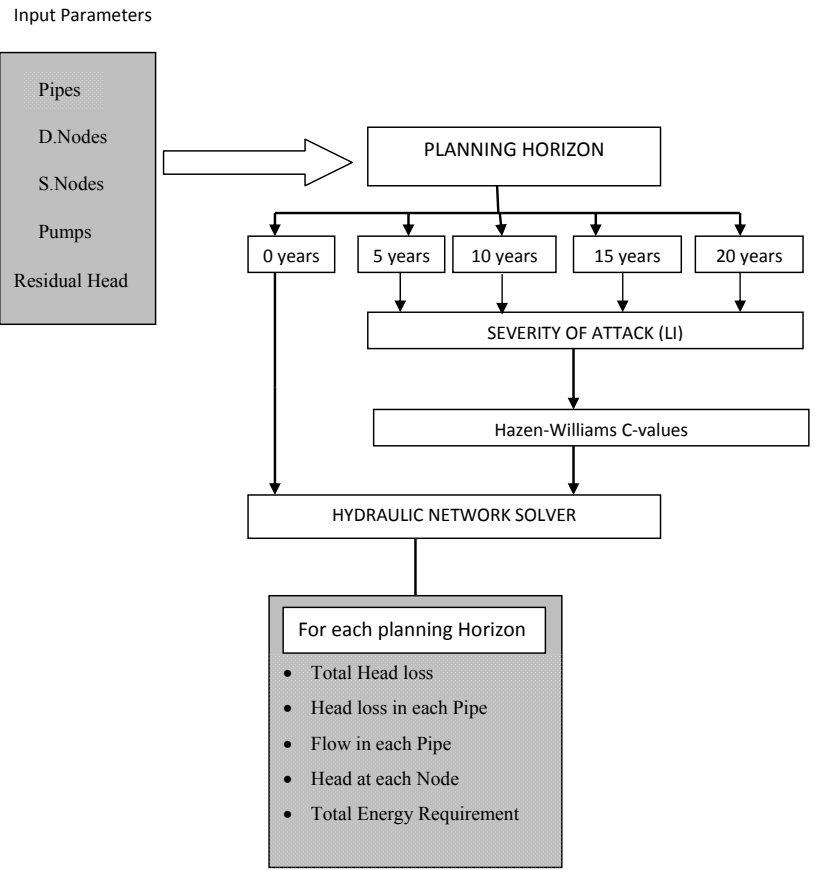

Figure 7. Schematic of planning module.

\subsection{Daily run module}

Many water utilities use human operators whose primary function is to monitor the pulse of the water distribution system and provide system control when needed. Automated control is increasingly becoming the preferred option and uses instrumentation and control equipment to control the distribution system automatically. Such control can be implemented either locally at the facility or throughout the system. Typically, simple operating rules are used to determine which component is operated and how. Systems that rely on advanced control use optimization algorithms, DSS, AI, or control logic to control the distribution system. It becomes clear that for this paradigm to be realistic, the actual system operation, in terms of its numerous diagnostic attributes, needs to be monitored continuously and logged for prospective reference. The proposed design of the DSS uses a Daily Run Module (DRM) that logs these operational attributes in order to be able to offer diagnostic inferences from the logged daily runs. Figure 9 depicts a selected screen shot of DRM. As shown in Fig. 8, this module has subordinate component sub-modules for the following tasks: (i) retrieve status of the study network on some previous date and time (Sub-ModuleI); (ii) generate daily run scenario for a given set of input conditions (Sub-Module-II); and (iii) diagnose specific consumer complaints (Sub-Module-III). A brief note on each of these sub-modules follows:

- Sub-Module-I: refer to Fig. 9 with dotted circle "I". Each daily run history, for a given set of input conditions, is stored in the knowledge base of the DSS. The 


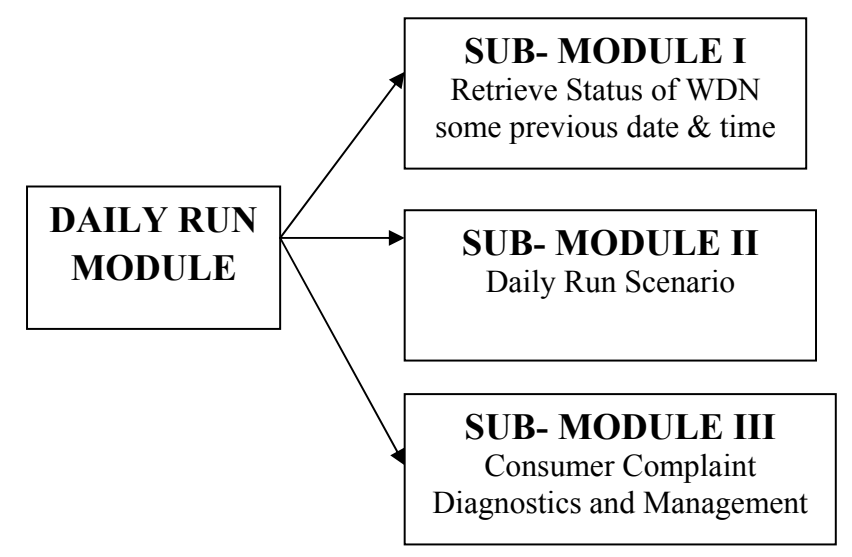

Figure 8. Different modules of Daily Run Module.

status of the WDN corresponding to any given date and time can be retrieved with the help of the graphical user interface (GUI) and runs independent of CLIPS inference engine.

- Sub-Module-II: refer to Fig. 9 with dotted circle "II". This sub-module assists a network manager in ascertaining status of the network corresponding to operator specified inputs on source node levels, pump, and electricity status. There is also an option available that allows comparison between observations on link flows and nodal heads, provided to the sub-module as prior specified data, and corresponding computed values as output from the network solver. The comparison is made by the inference engine of CLIPS, shown in Fig. 10, and is followed by generation of appropriate caution and/or advice. The inference engine fires rules at each decision stage, identifies cause, and also proposes action using the backward chaining mechanism. Figure 10 presents "if else" rules firing under different scenarios with message details. For example, if comparison of flows and heads with normal values is found OK, then message "No Action Required" is popped up; otherwise, "Rule 2" is fired and reservoir levels are compared.

- Sub-Module-III: This is the Daily Diagnostic Module and is designed to manage consumer complaints. The module uses backward chaining process for inferencing and starts by first identifying the consumer in terms of the network segment that he/she belongs to. Rules for this module are similar to those shown in Fig. 10. Using backward chaining mechanism, CLIPS is designed to identify possible cause of the complaint and, as a logical follow up, also suggests appropriate measures to be adopted to manage the situation.

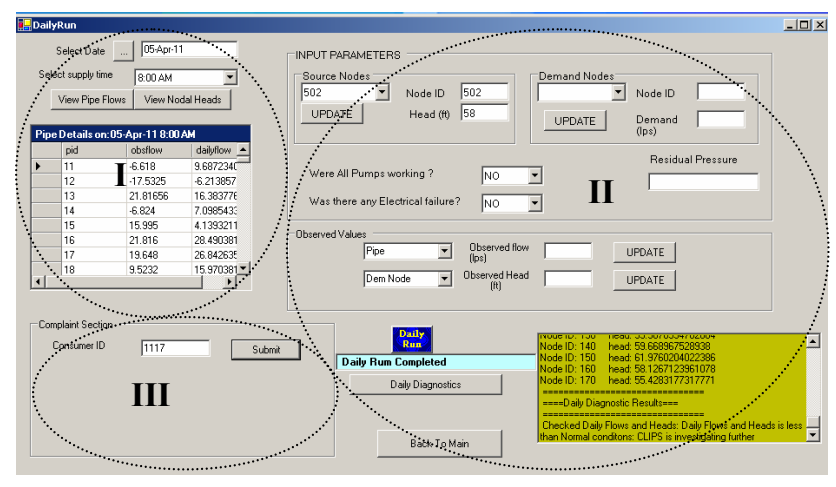

Figure 9. Screen shot of Daily Run Module of DSS showing submodules.

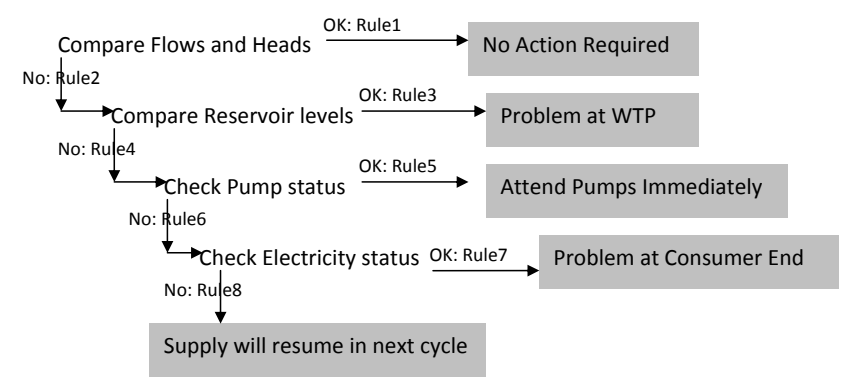

Figure 10. Working of CLIPS for Sub-Module II.

\section{Case study}

The proposed application has been implemented on an existing water distribution network that services a segment of Vasant Vihar (identified as Blocks "D", "E", and "F") in New Delhi. The study area is a predominantly residential area having a total population of 21000 . The network consists of 111 pipes and 86 demand nodes. Processing any new project on the proposed ES platform begins with data formatting and is done invoking the "New Network" stream of DSS. Figure 11 shows "New Project" form of DSS for formatting data pertaining to WDN.

By clicking "Add Base Map" button, the JPEG file of the desired network can be loaded on the form which would facilitate creation of shape files. Source Node, Demand Node, Pumps and Valves are added as nodes on the shape file "node.shp". A pipe is added as line in the shape file "pipe.shp". Upon conclusion of this task, the project network is then processed as "Existing Network" in DSS.

\subsection{Calibration}

The DSS adopts an optimization based approach for calibration and is enabled by the state-of-the-art ant colony optimization algorithm (ACO). As an added novelty, the calibration algorithm is also designed to accommodate prior 


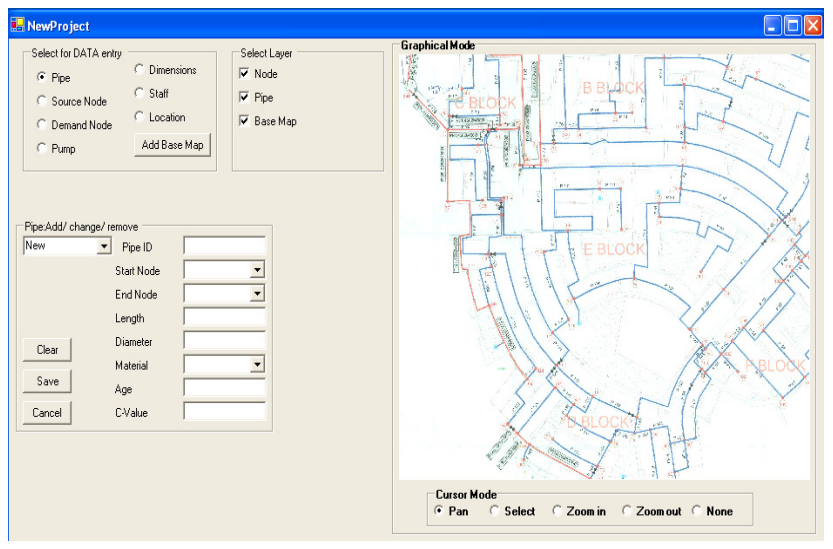

Figure 11. New Project form of ES.

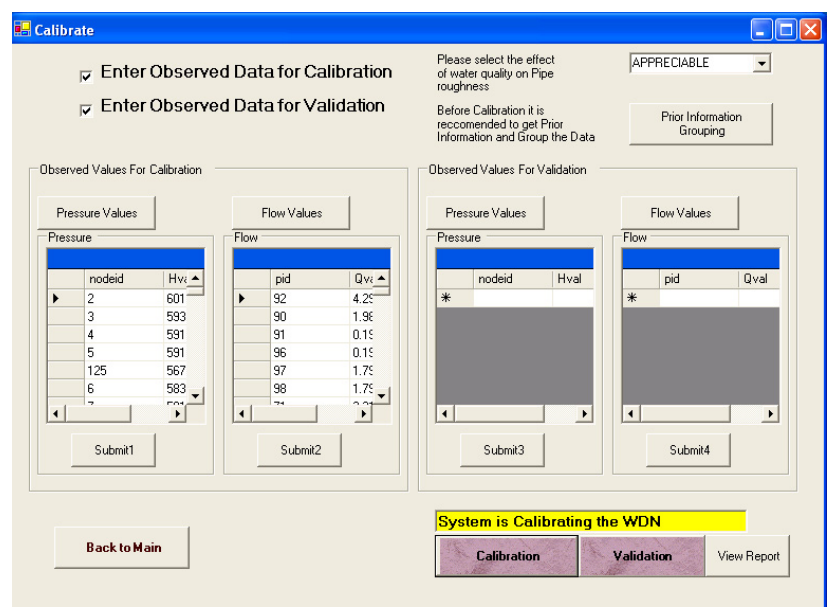

Figure 12. Screen Shot of the Calibration Module.

information on age of the network components as a guide leading the search process towards the desired optimality. As shown in Fig. 12, calibration is initiated by prompting the user to provide requisite information such as observed link flows and junction heads (these will form the basis for comparison between these values and model derived outputs).

Before proceeding for calibration, the module groups pipes based on diameter and age and computes prior information on Hazen-William C-values for each such group based on type of attack on the pipes on account of quality of water. The calibration module derives and assigns calibrated Cvalues to each pipe, and these results are archived as shown in Fig. 13

\subsection{Simulation}

The CLIPS engine has been designed to be an ideal foil for the simulation module and allows the DSS to exploit the full potential of the latter module. Each simulation outcome is checked for precedence, and only those scenarios that are

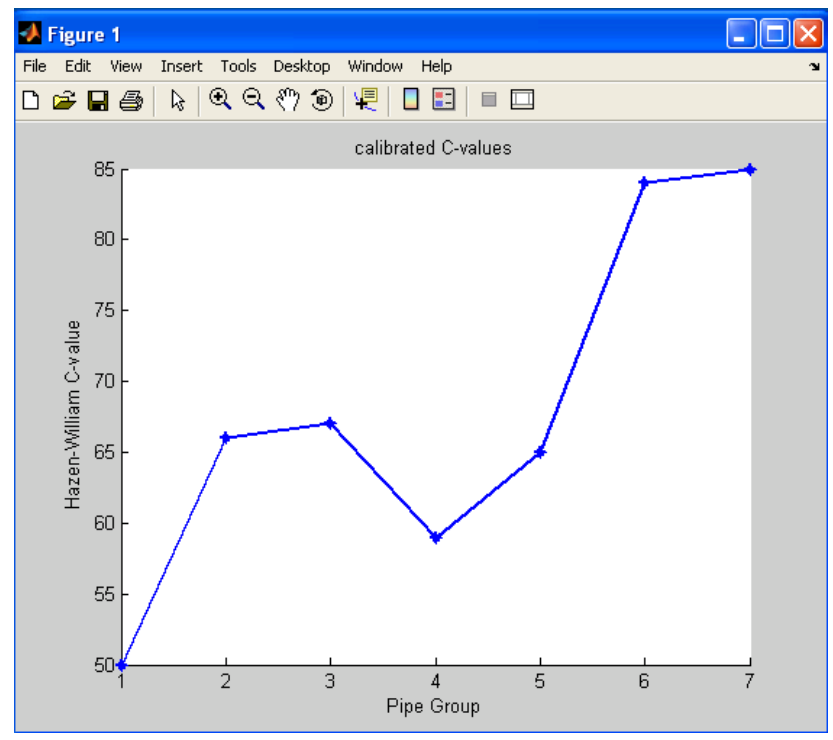

Figure 13. Calibration result for Vasant Vihar WDN.

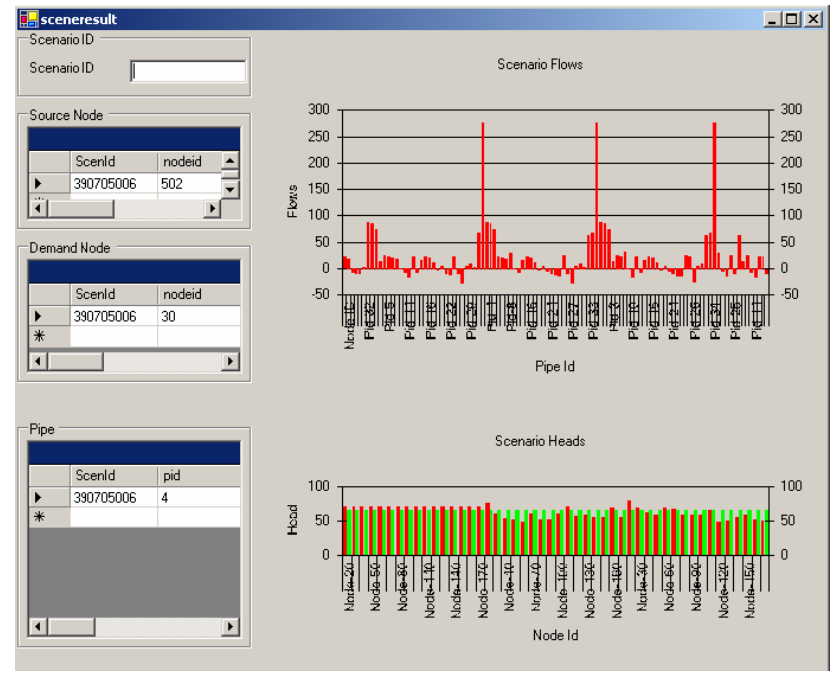

Figure 14. Existing scenario results of Simulation module.

not already available in the knowledge base are imbibed into it with a new, unique ID for possible future use. It is understood that the design of the proposed DSS has distinctive features such as: (i) the knowledge base (KB) is continuously upgraded; and (ii) the knowledge base is dynamic. Figure 14 presents a screen shot of the results propagated by simulation mModule in terms of link flows and nodal heads with the latter being displayed in comparison with residual head. Also, the left part of the figure displays changes in pipes, demand nodes, and source nodes that make up a particular scenario.

Simulation studies are undertaken to diagnose problems related to WDN. As an example, consider a scenario 1 in Fig. 15. Initially, source node 501 is maintained at reservoir level of $2.5 \mathrm{~m}$. At this condition head at node 20 was $8.90 \mathrm{~m}$. 


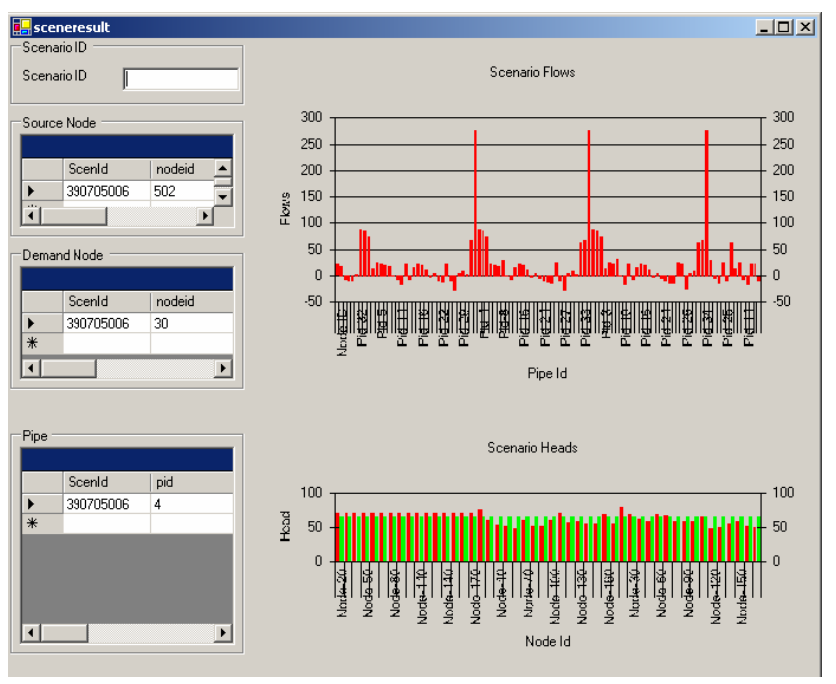

Figure 15. Scenario for problem identification and remedial measures.

Considering desirable residual head of $12.0 \mathrm{~m}$ at node 20 , network administrator proposes to change pipe diameters of pipe number 135 and 34 (feeding to node 20) from existing $100 \mathrm{~mm}$ to $200 \mathrm{~mm}$ and $150 \mathrm{~mm}$, respectively. Since infrastructure improvement work involves huge cost, the simulation module can be used to study the impact of such proposed changes. It can be seen from Fig. 15 that head at node 20 of Vasant Vihar WDN has improved to 13.61 (above residual head). The scenario can be saved for future reference. Whenever such scenario will be tried, the module shall issue a message "Similar scenario was earlier tried. Do you want to view the scenario?". If user chooses "Yes", then existing scenario details are populated; otherwise, scenario is processed as new scenario.

\subsection{Planning}

Planning module of the DSS has been designed to give an insight into the WDN's performance over planning horizon. Planning horizon has been taken as 20 years with a time step of 5 years (i.e. 5, 10, 15, and 20 years). The module is designed to help network managers take guidance on decisions for replacement of pipes and upgradation of pumping stations. In this regard, the module derives strength based on its ability to report the following:

- Variation of pipe flows and junction heads over the different planning horizon (Fig. 16).

- Variation of Hazen-William C-values for different groups of Pipes over the different planning horizon (Fig. 17).

- Variation of total energy requirement in $\mathrm{kW}$ and total head loss in the system over the different planning horizon (Fig. 18).

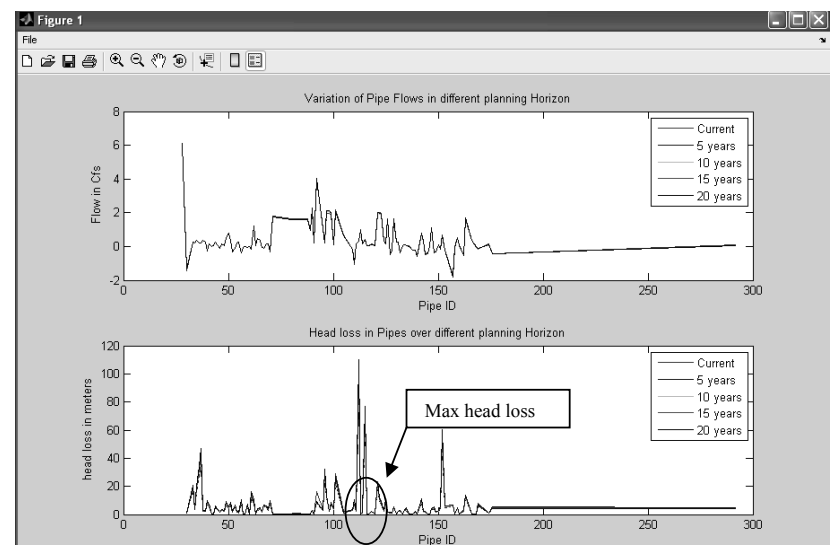

Figure 16. Variation of flows and heads over PH.

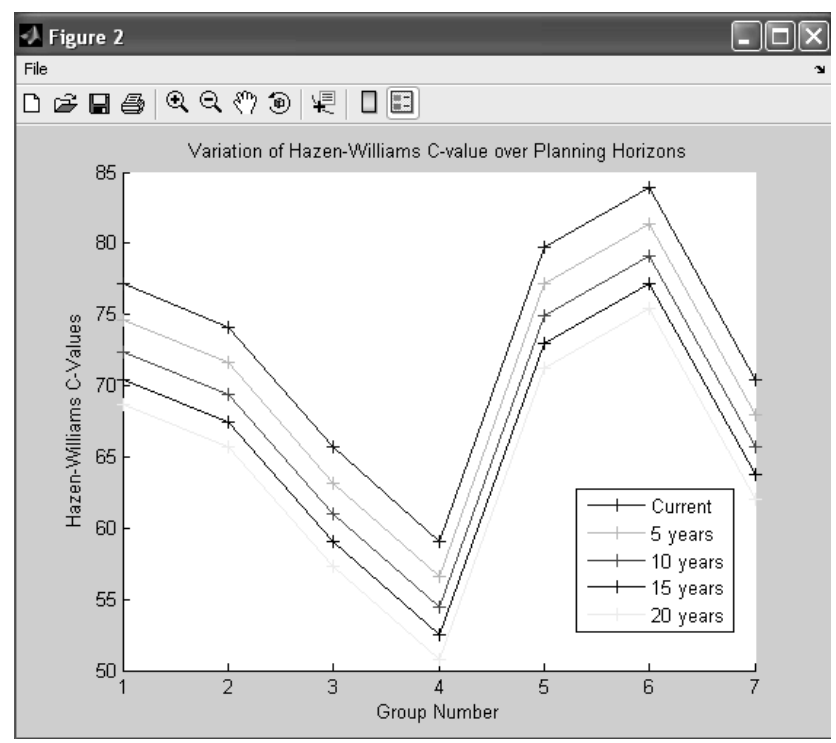

Figure 17. Variation of Hazen-William C-values for different groups.

\subsection{Daily run}

The DSS is built with a daily run module (DRM) and as already explained, this module consists of three sub-modules I, II, and III. Sub-Module-I prompts user to select date and supply time (for intermittent supply) for information on link flows and junction heads. For this already existing scenario, the module presents information as depicted in Fig. 8. The sub-modules II and III are tailored to invoke CLIPS inference engine to assert facts derived as a solution from the hydraulic network solver in its memory, and importantly, follow up with Advice/Suggestion/Warning as deemed appropriate as illustrated in Fig. 19.

The daily tun module is also endowed with an important functionality in terms of its ability to manage consumer 


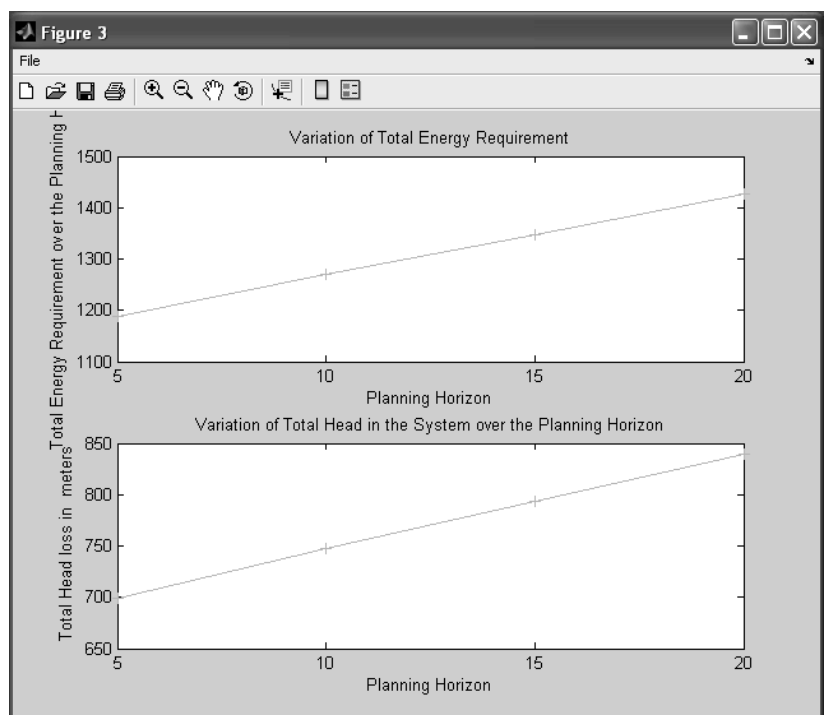

Figure 18. Variation of Total Energy and total head loss.

complaints. Consider, for example, the water reservoir (ID 501) of the case study. The following information is lodged:

1. Reported head at the reservoir (ID 501) is $2.5 \mathrm{~m}$ instead of $3.0 \mathrm{~m}$.

2. Consumer with ID 3423 lodges a complaint that "water supply to the user has been less than normal".

3. Reports of a pump failure have also been independently lodged.

4. There was no reported electricity failure.

From the network configuration, it is seen that the complainant is connected to pipe ID $71 \mathrm{u} / \mathrm{s}$ node 3 and $\mathrm{d} / \mathrm{s}$ node 30 ). The CLIPS inference engine uses backward chaining mechanism and reports appropriately reasoned diagnostic message as depicted in Fig. 20 which shows successive messages as would be received from the consumer complaint management module.

\section{Conclusions}

The aim of the development of DSS was to design a comprehensive operation and management aid for a water utility. The study has demonstrated the potential application of CLIPS as a core component around which such a management aid could be built. Present work suggests that CLIPS is indeed a powerful platform for development of an expert system.

Supporting platforms like MATLAB, SQL, and MapWinGIS have also been integrated together under a common umbrella of Visual Studio.Net environment. In this work, the

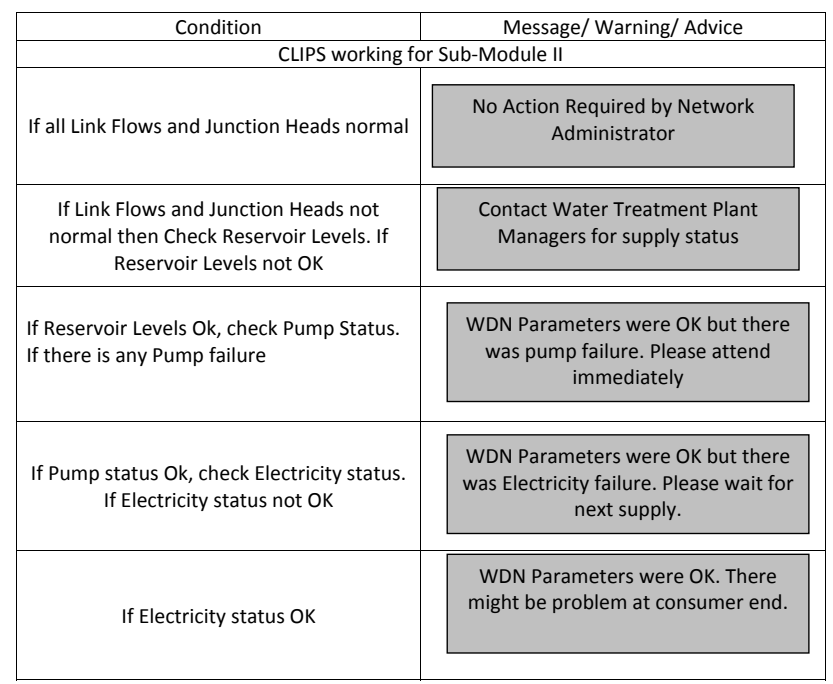

Figure 19. Advice/Suggestion/Warning issued by CLIPS.

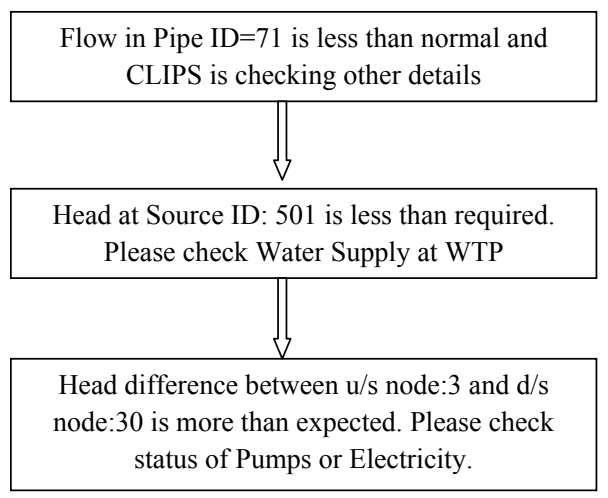

Figure 20. Messages received for addressing consumer complaint.

CLIPS inference engine has been linked directly with Visual Studio.Net environment and indirectly with SQL and MATLAB. Such interlinking of these seemingly diverse set of computing platforms has resulted in a multiplier effect and, together, the study has been able to unlock the combined potential of such an application. In particular, the proposed framework acquires a totally dynamic flavor where a water supply system can be modelled, calibrated, and validated and its hydraulic performance simulated for a diverse set of operating requirements. The modules of DSS, as designed, can then acquire facts that emerge from such an exercise for further analysis and post processing by the inference engine and its resident and equally dynamic and flexible knowledge base.

Furthermore, the proposed DSS has been designed to perform other routine tasks such as directed simulation runs in response to any real or hypothetical intervention and to assess the given study network for consequent impacts. This 
helps in a priori generation of knowledge regarding network performance following such interventions.

CLIPS platform does not have permanent memory and an important contribution of the study has been the development of an external dynamic knowledge base with the mechanism for transferring newer facts into the knowledge base for subsequent use by the inference engine of CLIPS. The dynamic knowledge domain is designed to be self learning as it autogenerates newer knowledge for the DSS for future guidance.

The DSS, with its simulation and planning modules, is able to estimate, in advance, the expected Hazen Williams C- values for planning horizons of 5, 10, 15, and 20 years. Importantly, the proposed DSS avoids excessive dependence on interventions by highly trained expert personnel and with the help of a highly user friendly interface, even relatively novice operators are now vested with necessary tools for effective management.

\section{Scope for further developments}

In order to enhance the appeal of the proposed DSS for water supply systems that are in continuous operation and the system is essentially driven by demand, Extended Period Simulation (EPS) would be a desirable addition. Further, in present day scenario, it would be advantageous to harness the potential of Information Technology in water sector. Most water utilities now use some form of Supervisory Control and Data Acquisition (SCADA) system in their daily operations. The DSS would be an ideal partner tool for a SCADA based water distribution management system and, undoubtedly, would significantly widen the utility of the proposed Expert System.

\section{Edited by: I. Worm}

\section{References}

Boulos, P. and Ormsbee, L.: Explicit network calibration for multiple loading conditions, Civ. Eng. Environ. Syst., 8, 153-160, 1991.

Bunn, S. and Helms, S.: Application of an expert system to control treated water distribution, ASCE, Annual Water Resource Planning and Management Conference, Phoenix, AZ, 1999.

Chan, C., Kritpiphat, W., and Tontiwachwuthikul, P.: Development of an intelligent control system for a municipal water distribution network, Proceedings of the 1999 IEEE Canadian Conference on Electrical and Computer Engineering, Edmonton, Alberta, Canada, 9-12 May 1999.

Chau, K.: Knowledge-based system on water-resource management in coastal waters, Water Environ. J., 18, 25-28, 2004.

Cheng, C. T., Ou, C. P., and Chau, K. W.: Combining a fuzzy optimal model with a genetic algorithm to solve multi-objective rainfall-runoff model calibration, J. Hydrol., 268, 72-86, 1999.

Coulbeck, B. and Orr, C.: A network analysis and simulation program for water distribution systems, Civ. Eng. Environ. Syst., 1, 139-144, 1984.
Colebrook, C. and White, C.: The reduction of carrying capacity of pipes with age, J. Ice, 7, 99-118, 1937.

CAC - Calibration Guidelines for Water Distribution System Modelling: Engineering Computer Application Committee, Proceedings of AWWA 1999 1mTech conference, American Water Works Association, 1999.

Gofman, E. and Rodeh, M.: Loop equations with unknown pipe characteristics, J. Hydraul. Div., 107, 1047-1060, 1982.

Goforth, G. and Floris, V.: OASIS: An intelligent water management system for South Florida, AI Appl., 5, 47-55, 1991.

Kapelan, Z.: Calibration of water distribution system hydraulic models, PhD thesis, University of Exeter, 2002.

Khosa, R., Parida, B., Singh, B., and Aggarwal, S.: Expert system for iit water supply, Master's thesis, Unpublished Undergraduate Project under Student Undergraduate Research Award(SURA), Indian Institute of Technology, Delhi, 1995.

Kulshrestha, S., Khosa, R., and Jha, K.: Roughness Calibration of a Water Distribution Network using Ant Colony Optimization with Prior Information, J. Water Resour. Pl.-ASCE, in review, 2001.

Lamont, P.: Common pipe flow formulas compared with the theory of roughness, J. Am. Water Work. Assoc., 73, 274-280, 1981.

Leon, C., Martín, S., Elena, J., and Luque, J.: EXPLORE- hybrid expert system for water networks management, J. Water Resour. Pl.-ASCE, 126, 65-74, 2000.

Machell, J., Mounce, S. R., and Boxall, J. B.: Online modelling of water distribution systems: a UK case study, Drink. Water Eng. Sci., 3, 21-27, doi:10.5194/dwes-3-21-2010, 2010.

Maier, H., Simpson, A., Zecchin, A., Foong, W., Phang, K., Seah, H., and Tan, C.: Ant colony optimization for design of water distribution systems, J. Water Resour. Pl.-ASCE, 129, 200-209, 2003.

Muttil, N. and Chau, K. W.: Machine-learning paradigms for selecting ecologically significant input variables, Eng. Appl. Artif. Intell., 20, 735-744, 2007.

Ormsbee, L.: Implicit network calibration, J. Water Resour. Pl.ASCE, 115, 243-257, 1989.

Ormsbee, L. and Wood, D.: Explicit pipe network calibration, J. Water Resour. Pl.-ASCE, 112, 166-182, 1986.

Raghavendran, V., Gonsalves, T. A., Rani, U., Kumar, S., and Narasimhan, S.: Design and Implementation of a Network Management System for Water Distribution Networks, ADCOM'07 Proceedings of the 15th International Conference on Advanced Computing and Communications, IEEE Computer Society Washington, DC, USA, doi:10.11.09/ADCOM.2007.62, 2007.

Rahal, C., Sterling, M., and Coulbeck, B.: Parameter tuning for simulation models of water distribution networks, Proc. Inst. Eng., 69, 751-762, 1980.

Rossman, L.: EPANET water quality model, Technical report, PB93-236552/XAB, Environmental Protection Agency, Cincinnati, OH (United States), Risk Reduction Engineering Lab., 1993.

Shepherd, A. and Ortolano, L.: Water-supply system operations: Critiquing expert-system approach, J. Water Resour. Pl.-ASCE, 122, 348-355, 1996.

Swamee, P. and Jain, A.: Explicit equation for pipe-flow problems, ASCE J. Hydraul. Div., 102, 657-664, 1976.

Todini, E. and Pilati, S.: Gradient Algorithm for the Analysis of Pipe Networks, Computer Applications in Water Supply, 1, Research Studies Press Ltd. Taunton, UK, 1988. 
Walski, T.: Technique for calibrating network models, J. Water Resour. Pl.-ASCE, 109, 360-372, 1983.

Walski, T.: Tips for saving energy in pumping operations, J. Am. Water Work. Assoc., 85, 49-53, 1993.

Walski, T., Shields, F., and Sharp, W.: Predicting Internal Roughness in Water Mains, Miscellaneous Paper EL-88-2, US Army Engineer Waterways Experiment Station, Vicksburg, 1988.

Walski, T., Chase, D., Savic, D., Strafaci, A., and Dietrich, K.: Water Distribution Modelling, Haestad Press, Waterbury, CT, USA, 2001.
Wang, H. J., Zhang, J. P., Chau, K. W., and Anson, M.: 4D dynamic management for construction planning and resource utilization, Automat. Construct., 13, 575-589, 2004.

Zecchin, A., Simpson, A., Maier, H., and Nixon, J.: Parametric study for an ant algorithm applied to water distribution system optimization, IEEE T. Evol. Comput., 9, 175-191, 2005. 